\title{
Monitoring Mortality Caused by COVID-19 Using Gamma- Distributed Variables Based on Generalized Multiple Dependent State Sampling
}

\author{
Muhammad Aslam $\mathbb{D},{ }^{1}$ G. Srinivasa Rao $\mathbb{D},{ }^{2}$ Muhammad Saleem $\mathbb{D},{ }^{3}$ \\ Rehan Ahmad Khan Sherwani, ${ }^{4}$ and Chi-Hyuck Jun ${ }^{5}$ \\ ${ }^{1}$ Department of Statistics, Faculty of Science, King Abdulaziz University, Jeddah 21551, Saudi Arabia \\ ${ }^{2}$ Department of Mathematics and Statistics, CNMS, The University of Dodoma, Dodoma, PO Box: 259, Tanzania \\ ${ }^{3}$ Department of Industrial Engineering, Faculty of Engineering-Rabigh, King Abdulaziz University, Jeddah 21589, Saudi Arabia \\ ${ }^{4}$ College of Statistical and Actuarial Sciences, University of the Punjab Lahore, Pakistan \\ ${ }^{5}$ Department of Industrial and Management Engineering, POSTECH, Pohang 790-784, Republic of Korea
}

Correspondence should be addressed to Muhammad Aslam; aslam_ravian@hotmail.com

Received 31 December 2020; Revised 15 March 2021; Accepted 8 April 2021; Published 29 April 2021

Academic Editor: Nadia A. Chuzhanova

Copyright (c) 2021 Muhammad Aslam et al. This is an open access article distributed under the Creative Commons Attribution License, which permits unrestricted use, distribution, and reproduction in any medium, provided the original work is properly cited.

More recently in statistical quality control studies, researchers are paying more attention to quality characteristics having nonnormal distributions. In the present article, a generalized multiple dependent state (GMDS) sampling control chart is proposed based on the transformation of gamma quality characteristics into a normal distribution. The parameters for the proposed control charts are obtained using in-control average run length (ARL) at specified shape parametric values for different specified average run lengths. The out-of-control ARL of the proposed gamma control chart using GMDS sampling is explored using simulation for various shift size changes in scale parameters to study the performance of the control chart. The proposed gamma control chart performs better than the existing multiple dependent state sampling (MDS) based on gamma distribution and traditional Shewhart control charts in terms of average run lengths. A case study with real-life data from ICU intake to death caused by COVID-19 has been incorporated for the realistic handling of the proposed control chart design.

\section{Introduction}

One of the important techniques for improving manufactured product quality and for reducing the manufacturing costs is statistical quality control (SQC). Since the pioneer work by Shewhart A. Walter during 1920s in Bell Telephone Laboratories, wide varieties of control chart techniques have been constructed and extensively implemented in SQC. The main feature of control charting is to identify the amount of assignable cause(s) and hence rectify it by taking necessary action on the production process before sending the outcome of the products into the market. This control charting helps to avoid nonconforming products from being manufactured by the company. More details about Shewhart control charts can be seen in Montgomery [1].
Usually, control charts are being designed and operating under the assumption of the normality for the variable of interest. Nevertheless, these assumptions may not be true for various realistic situations and other distributions away from normality had been considered and discussed by many authors in the literature (e.g., see [2-5]). The waiting time of an event, for example, can be represented by a gamma distribution as in [6]. Numerous researchers concentrate on quality characteristic understudy which follows a nonnormal distribution or transformed into normality to apply Shewhart type control charts. For skewed data, the gamma distribution is widely used. The works on the control charts for the gamma distribution are presented by Al-Oraini and Rahim [7], Jearkpaporn et al. [8], Sheu and Lin [9], Aslam et al. [10], and Zhang et al. [11]. Santiago and Smith [5] used 
transformation given by Johnson and Kotz [12] and Nelson [13]. Mohammed [14], Mohammed and Laney [15], and Aslam et al. [16] discussed the application of the $t$-chart.

Several researchers have developed diversified sampling designs to obtain more efficient control charts. Recently, researchers focused on multiple dependent state (MDS) sampling in the creation of a control chart. Wortham and Baker [17] proposed the MDS sampling in quality control charts. MDS design is more competent than the existing single sampling plans because it considers the previous lot information along with the current lot to make a decision whether the process is under control or not (see [18]). Aslam et al. [19] developed a control chart for gamma distribution using MDS sampling. The control chart scheme using MDS sampling was studied by different authors for various schemes (see $[16,19,20-29,30,31]$ ).

More recently, Raza and Aslam [32], Rao et al. [33], Rao et al. [34], and Aslam et al. [35, 36] formulated generalized MDS (GMDS) sampling for various schemes. GMDS is more flexible and efficient than MDS to design the control chart using the gamma distribution. The aim of this article is to construct a gamma control chart for monitoring the process mean based on GMDS sampling design. The application of the proposed chart will be given using the COVID-19 mortality data. It is expected that the proposed chart will perform better than the existing Shewhart control chart and control charts using MDS in terms of average run length and standard deviation of run length.

\section{Design of Control Chart for Gamma Distribution Based on GMDS Sampling}

The proposed control chart for a gamma distribution using gamma to normal transformation is discussed. Let $X$ be a random variable from a gamma distribution with shape parameter $\alpha$ and scale parameter $\beta$. The cumulative distribution function (cdf) of the gamma distribution is given by

$$
p(X \leq x)=1-\sum_{k=0}^{\alpha-1} \frac{e^{-x / \beta}(x / \beta)^{k}}{k !} .
$$

Wilson and Hilferty [37] recommended that if $X$ follows a gamma distribution with specific parameters, then the transformed variable $X^{*}=X^{1 / 3}$ can be distributed approximately as normal with mean $\mu_{X^{*}}$ and variance $\sigma_{X^{*}}^{2}$, where

$$
\begin{aligned}
& \mu_{X^{*}}=\frac{\beta^{1 / 3} \Gamma(\alpha+1 / 3)}{\Gamma \alpha}, \\
& \sigma_{X^{*}}^{2}=\frac{\beta^{2 / 3} \Gamma(\alpha+2 / 3)}{\Gamma \alpha}-\mu_{X^{*}}^{2} .
\end{aligned}
$$

The proposed gamma control chart using GMDS sampling comprises the two pairs of control chart limits. The inner lower control limit (LCL) and upper control limit
(UCL) are denoted by subscript 1 , and the outer lower control limit (LCL) and upper control limit (UCL) are denoted by subscript 2 . The four control limits are given by

$\mathrm{UCL}_{1}=\mu_{X^{*}}+k_{1} \sigma_{X^{*}}=\frac{\beta^{1 / 3} \Gamma(\alpha+1 / 3)}{\Gamma \alpha}+k_{1} \sqrt{\frac{\beta^{2 / 3} \Gamma(\alpha+2 / 3)}{\Gamma \alpha}-\mu_{X^{*}}^{2}}$

$\mathrm{LCL}_{1}=\mu_{X^{*}}-k_{1} \sigma_{X^{*}}=\frac{\beta^{1 / 3} \Gamma(\alpha+1 / 3)}{\Gamma \alpha}-k_{1} \sqrt{\frac{\beta^{2 / 3} \Gamma(\alpha+2 / 3)}{\Gamma \alpha}-\mu_{X^{*}}^{2}}$,

$\mathrm{UCL}_{2}=\mu_{X^{*}}+k_{2} \sigma_{X^{*}}=\frac{\beta^{1 / 3} \Gamma(\alpha+1 / 3)}{\Gamma \alpha}+k_{2} \sqrt{\frac{\beta^{2 / 3} \Gamma(\alpha+2 / 3)}{\Gamma \alpha}-\mu_{X^{*}}^{2},}$

$\mathrm{LCL}_{2}=\mu_{X^{*}}-k_{2} \sigma_{X^{*}}=\frac{\beta^{1 / 3} \Gamma(\alpha+1 / 3)}{\Gamma \alpha}-k_{2} \sqrt{\frac{\beta^{2 / 3} \Gamma(\alpha+2 / 3)}{\Gamma \alpha}-\mu_{X^{*}}^{2},}$

where $k_{1}$ and $k_{2}$ are the chart constants to be found when the in-control ARL is approximately equal to preassigned value $r_{0}$. The convenient form of the above control limits is given as follows: $\mathrm{UCL}_{1}=\beta^{1 / 3} \mathrm{UL}_{1}, \mathrm{UCL}_{2}=\beta^{1 / 3} \mathrm{UL}_{2}, \mathrm{LCL}_{1}=\beta^{1 / 3}$ $\mathrm{LL}_{1}$, and $\mathrm{LCL}_{2}=\beta^{1 / 3} \mathrm{LL}_{2}$, where

$$
\begin{aligned}
& \mathrm{LL}_{1}=\frac{\Gamma(\alpha+1 / 3)}{\Gamma \alpha}-k_{1} \sqrt{\frac{\Gamma(\alpha+2 / 3)}{\Gamma \alpha}-\left(\frac{\Gamma(\alpha+1 / 3)}{\Gamma \alpha}\right)^{2}}, \\
& \mathrm{LL}_{2}=\frac{\Gamma(\alpha+1 / 3)}{\Gamma \alpha}-k_{2} \sqrt{\frac{\Gamma(\alpha+2 / 3)}{\Gamma \alpha}-\left(\frac{\Gamma(\alpha+1 / 3)}{\Gamma \alpha}\right)^{2}}, \\
& \mathrm{UL}_{1}=\frac{\Gamma(\alpha+1 / 3)}{\Gamma \alpha}+k_{1} \sqrt{\frac{\Gamma(\alpha+2 / 3)}{\Gamma \alpha}-\left(\frac{\Gamma(\alpha+1 / 3)}{\Gamma \alpha}\right)^{2}}, \\
& \mathrm{UL}_{2}=\frac{\Gamma(\alpha+1 / 3)}{\Gamma \alpha}+k_{2} \sqrt{\frac{\Gamma(\alpha+2 / 3)}{\Gamma \alpha}-\left(\frac{\Gamma(\alpha+1 / 3)}{\Gamma \alpha}\right)^{2}} .
\end{aligned}
$$

The operation of the proposed control chart using GMDS scheme is described as follows:

(1) Obtain quality measurement from the manufacturing process, and denote the quality characteristic by $X$. Compute the transformed variable $X^{*}$ as $X^{*}=X^{1 / 3}$

(2) The process can be considered under control if $\mathrm{LCL}_{2} \leq X^{*} \leq \mathrm{UCL}_{2}$, and the process can be considered out-of-control if $X^{*} \geq \mathrm{UCL}_{1}$ or $X^{*} \leq \mathrm{LCL}_{1}$. Or else, go to Step 3 
(3) The process can be considered under control whenever $k$ out of $m$ proceeding subgroups have been declared as under control, that is, $\mathrm{LCL}_{2} \leq X^{*} \leq$ $\mathrm{UCL}_{2}$; otherwise, the output of the product can be considered out-of-control and go back to Step 1

The probability of declaring as in-control for the proposed control chart when the process is actually in-control is given as follows:

$$
P_{\text {in } .0}=P_{a .0}+P_{s .0}\left[\sum_{j=k}^{m}\left(\begin{array}{c}
m \\
j
\end{array}\right) P_{a .0}^{j}\left(1-P_{a .0}\right)^{m-j}\right],
$$

where

$$
\begin{aligned}
P_{a .0}= & p\left(\mathrm{LCL}_{2} \leq X^{*} \leq \mathrm{UCL}_{2} \mid \beta=\beta_{0}\right) \\
= & p\left(X^{*} \leq \mathrm{UCL}_{2} \mid \beta=\beta_{0}\right)-p\left(X^{*} \leq \mathrm{LCL}_{2} \mid \beta=\beta_{0}\right) \\
= & \sum_{k=0}^{\alpha-1} \frac{e^{-\mathrm{LL}_{2}^{3}\left(\mathrm{LL}_{2}^{3}\right)^{k}}}{k !}-\sum_{k=0}^{\alpha-1} \frac{e^{-\mathrm{UL}_{2}^{3}\left(\mathrm{UL}_{2}^{3}\right)^{k}}}{k !} \\
P_{s .0}= & p\left(\mathrm{LCL}_{1} \leq X^{*} \leq \mathrm{LCL}_{2} \mid \beta=\beta_{0}\right)+p\left(\mathrm{UCL}_{2}\right. \\
\leq & \left.X^{*} \leq \mathrm{UCL}_{1} \mid \beta=\beta_{0}\right) \\
= & \sum_{k=0}^{\alpha-1} \frac{e^{-\mathrm{LL}_{1}^{3}\left(\mathrm{LL}_{1}^{3}\right)^{k}}}{k !}-\sum_{k=0}^{\alpha-1} \frac{e^{-\mathrm{LL}_{2}^{3}\left(\mathrm{LL}_{2}^{3}\right)^{k}}}{k !} \\
& +\sum_{k=0}^{\alpha-1} \frac{e^{-\mathrm{UL}_{2}^{3}}\left(\mathrm{UL}_{2}^{3}\right)^{k}}{k !}-\sum_{k=0}^{\alpha-1} \frac{e^{-\mathrm{UL}_{1}^{3}}\left(\mathrm{UL}_{1}^{3}\right)^{k}}{k !}
\end{aligned}
$$

Therefore, the in-control average run length (ARL) when the process is under control is given by

$$
\mathrm{ARL}_{0}=\frac{1}{1-P_{\text {in. } 0}}
$$

Assume the gamma scale parameter has been changed from $\beta=\beta_{0}$ to $\beta=\beta_{1}=s \beta_{0}$, where $s$ is the shift value.

The probability of process is declared as in-control while the scale parameter which has been changed can be obtained as follows:

$$
P_{\text {in. } 1}=P_{a .1}+P_{s .1}\left[\sum_{j=k}^{m}\left(\begin{array}{c}
m \\
j
\end{array}\right) P_{a .1}^{j}\left(1-P_{a .1}\right)^{m-j}\right],
$$

where

$$
\begin{aligned}
P_{a .1} & =p\left(\mathrm{LCL}_{2} \leq X^{*} \leq \mathrm{UCL}_{2} \mid \beta=\beta_{1}\right) \\
& =p\left(X^{*} \leq \mathrm{UCL}_{2} \mid \beta=\beta_{1}\right)-p\left(X^{*} \leq \mathrm{LCL}_{2} \mid \beta=\beta_{1}\right) \\
& =\sum_{k=0}^{\alpha-1} \frac{e^{-\mathrm{LL}_{2}^{3} / s}\left(\mathrm{LL}_{2}^{3} / s\right)^{k}}{k !}-\sum_{k=0}^{\alpha-1} \frac{e^{-\mathrm{UL}_{2}^{3} / s}\left(\mathrm{UL}_{2}^{3} / s\right)^{k}}{k !}
\end{aligned}
$$

$$
\begin{aligned}
P_{s .1}= & p\left(\mathrm{LCL}_{1} \leq X^{*} \leq \mathrm{LCL}_{2} \mid \beta=\beta_{1}\right) \\
& +p\left(\mathrm{UCL}_{2} \leq X^{*} \leq \mathrm{UCL}_{1} \mid \beta=\beta_{1}\right) \\
= & \sum_{k=0}^{\alpha-1} \frac{e^{-\mathrm{LL}_{1}^{3} / s}\left(\mathrm{LL}_{1}^{3} / s\right)^{k}}{k !}-\sum_{k=0}^{\alpha-1} \frac{e^{-\mathrm{LL}_{2}^{3} / s}\left(\mathrm{LL}_{2}^{3} / s\right)^{k}}{k !} \\
& +\sum_{k=0}^{\alpha-1} \frac{e^{-\mathrm{UL}_{2}^{3} / s}\left(\mathrm{UL}_{2}^{3} / s\right)^{k}}{k !}-\sum_{k=0}^{\alpha-1} \frac{e^{-\mathrm{UL}_{1}^{3} / s}\left(\mathrm{UL}_{1}^{3} / s\right)^{k}}{k !} .
\end{aligned}
$$

The out-of-control average run length (ARL) when the process is out-of-control is given as

$$
\mathrm{ARL}_{1}=\frac{1}{1-P_{\text {in.1 }}}
$$

The proposed control chart parameters $k_{1}$ and $k_{2}$ along with ARL are obtained using the following algorithm: 1

(1) Decide the predetermined in-control ARL as $r_{0}$

(2) Fix the known values for $m$ and $k$

(3) Obtain the $\mathrm{ARL}_{0}$ using Equation (14), which consists of chart parameters $k_{1}$ and $k_{2}$

(4) Determine the most possible values of chart parameters $k_{1}$ and $k_{2}$ such that, $\mathrm{ARL}_{0} \geq r_{0}$

(5) In the above step, we get more values of $k_{1}$ and $k_{2}$ to satisfy the condition. Choose the best values of $k_{1}$ and $k_{2}$ for which the value of $\mathrm{ARL}_{0}$ is almost equal to $r_{0}$

(6) Using the best parametric values of $k_{1}$ and $k_{2}$ determined in the previous step, work out the $\mathrm{ARL}_{1}$ using Equation (18) and hence obtain standard deviation (SD) of run-length (SDRL) for various shift $(s)$ values

The $\mathrm{R}$ codes to find the design parameters of the control chart are given in the appendix.

\section{Numerical Results and Discussion}

The performance of the proposed gamma control chart using GMDS sampling is considered based on ARL, such as $\mathrm{ARL}_{0}$ and $A_{R L}$. These ARL values are used to know the effectiveness of the developed control chart. The developed chart is said to be efficient if it shows larger in-control ARL and smaller out-of-control ARL. Using the aforementioned algorithm in Section 2, the chart coefficients $k_{1}$ and $k_{2}$ are obtained. The out-of-control ARLs and SDRL are computed for a choice of shift values, $s$ from 1.0 to 2.0 with an interval of 0.1 and 2.0 to 4.0 with an interval of 0.5 . The values of $m$ considered are 4,5 , and 6 and $\alpha_{0}=5,10$, and 20. Table 1 is for $r_{0}=370$ and $\alpha_{0}=5$, Table 2 is for $r_{0}=370$ and $\alpha_{0}=10$, Table 3 is for $r_{0}=370$ and $\alpha_{0}=20$, Table 4 is for $r_{0}=500$ and $\alpha_{0}=5$, Table 5 is for $r_{0}=500$ and $\alpha_{0}=10$, and Table 6 is for $r_{0}=500$ and $\alpha_{0}=20$.

We pointed out the following several noteworthy comments from Tables 1-6 for the developed control charts: 







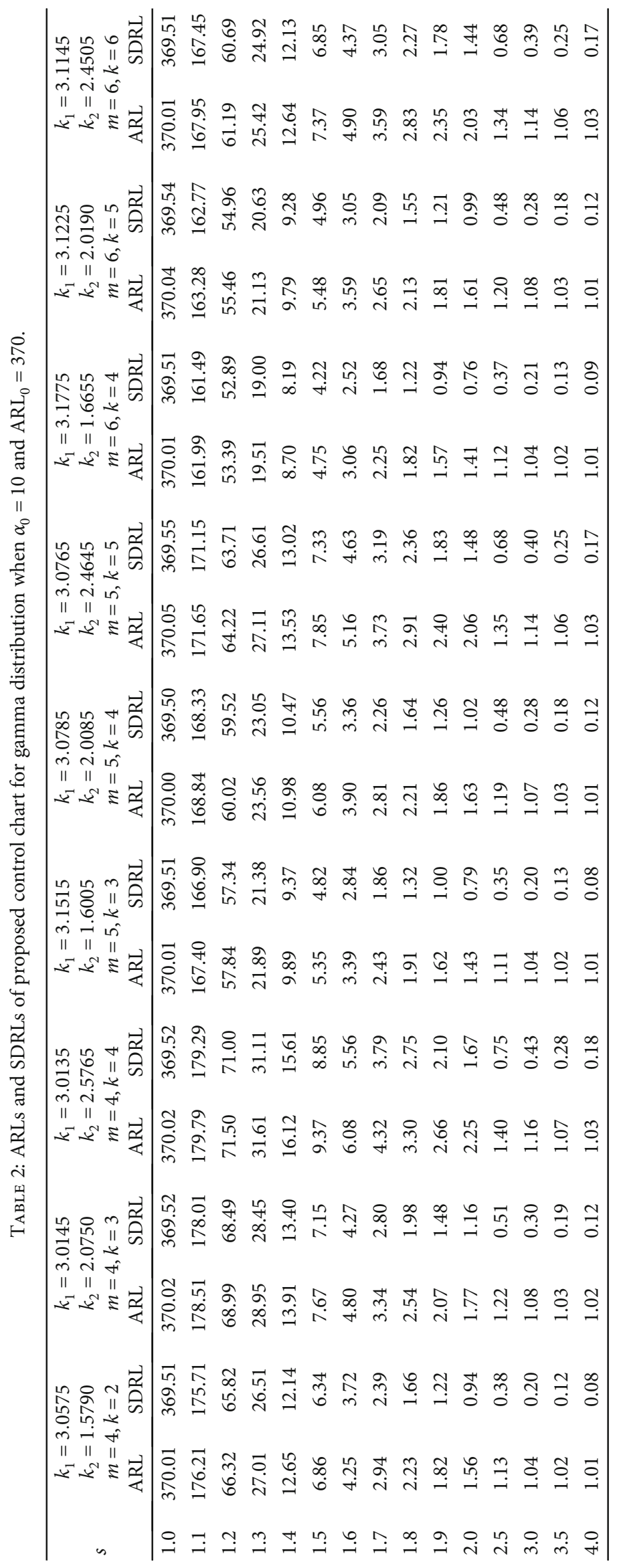




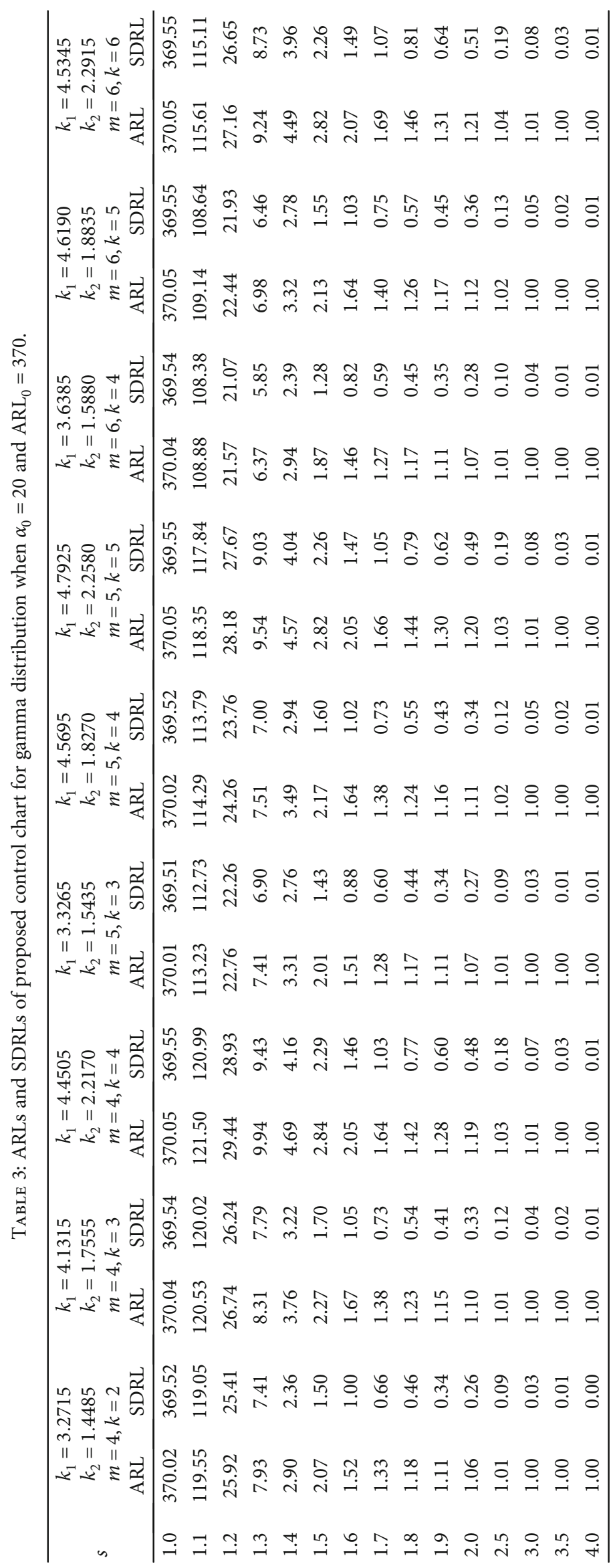









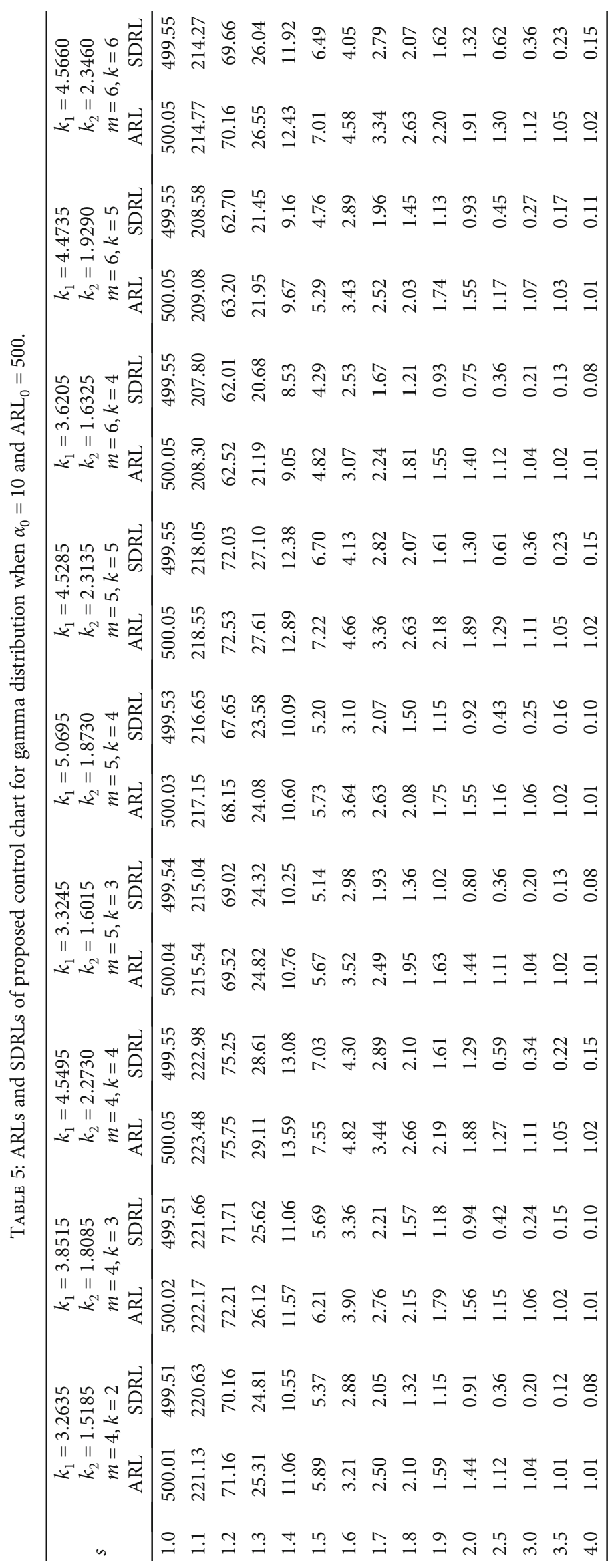




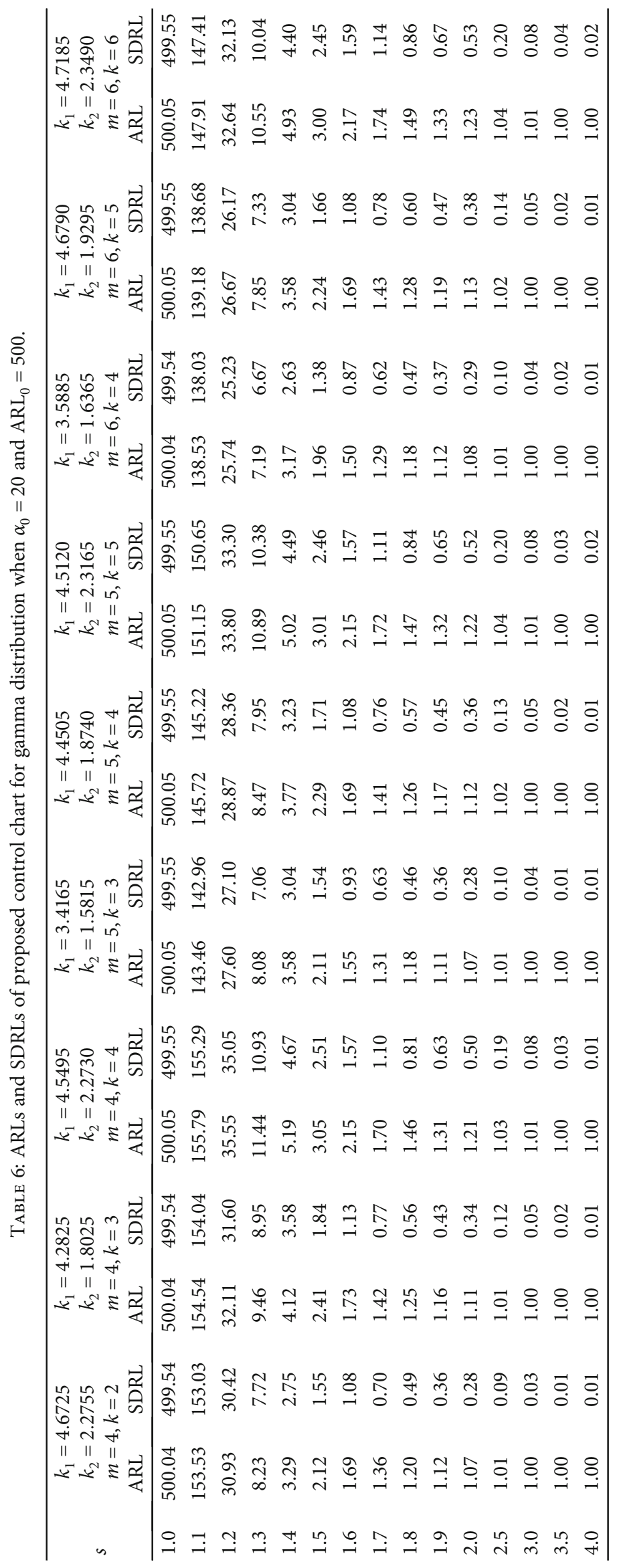


TABLE 7: Developed gamma control chart ARL comparison with existing control charts when $\mathrm{ARL}_{0}=370$ and $m=4$.

\begin{tabular}{|c|c|c|c|c|c|c|c|c|c|}
\hline$s$ & $\begin{array}{c}\text { Proposed } \\
k_{1}=3.1125 \\
k_{2}=1.5025\end{array}$ & $\begin{array}{c}\alpha_{0}=5 \\
\text { MDS } \\
k_{1}=3.0025 \\
k_{2}=2.5235\end{array}$ & $\begin{array}{c}\text { Shewhart } \\
L=2.9605\end{array}$ & $\begin{array}{c}\text { Proposed } \\
k_{1}=3.0575 \\
k_{2}=1.5790\end{array}$ & $\begin{array}{c}\alpha_{0}=10 \\
\text { MDS } \\
k_{1}=3.0135 \\
k_{2}=2.5765\end{array}$ & $\begin{array}{c}\text { Shewhart } \\
L=2.9821\end{array}$ & $\begin{array}{c}\text { Proposed } \\
k_{1}=3.2715 \\
k_{2}=1.4485\end{array}$ & $\begin{array}{c}\alpha_{0}=20 \\
\text { MDS } \\
k_{1}=4.4505 \\
k_{2}=2.2170\end{array}$ & $\begin{array}{c}\text { Shewhart } \\
L=2.9917\end{array}$ \\
\hline 1.0 & 370.05 & 370.02 & 370.96 & 370.01 & 370.02 & 370.96 & 370.02 & 370.05 & 370.96 \\
\hline 1.1 & 206.61 & 208.25 & 217.16 & 176.21 & 179.79 & 188.41 & 119.55 & 121.50 & 144.07 \\
\hline 1.2 & 100.36 & 104.12 & 114.73 & 66.32 & 71.50 & 80.54 & 25.92 & 29.44 & 46.78 \\
\hline 1.3 & 50.23 & 54.56 & 63.78 & 27.01 & 31.61 & 38.47 & 7.93 & 9.94 & 19.11 \\
\hline 1.4 & 27.17 & 31.10 & 38.44 & 12.65 & 16.12 & 20.97 & 2.90 & 4.69 & 9.59 \\
\hline 1.5 & 16.00 & 19.27 & 24.98 & 6.86 & 9.37 & 12.77 & 2.07 & 2.84 & 5.64 \\
\hline 1.6 & 10.21 & 12.87 & 17.30 & 4.25 & 6.08 & 8.50 & 1.52 & 2.05 & 3.75 \\
\hline 1.7 & 7.00 & 9.15 & 12.64 & 2.94 & 4.32 & 6.07 & 1.33 & 1.64 & 2.73 \\
\hline 1.8 & 5.11 & 6.87 & 9.64 & 2.23 & 3.30 & 4.60 & 1.18 & 1.42 & 2.15 \\
\hline 1.9 & 3.93 & 5.39 & 7.63 & 1.82 & 2.66 & 3.64 & 1.11 & 1.28 & 1.78 \\
\hline 2.0 & 3.16 & 4.40 & 6.22 & 1.56 & 2.25 & 3.00 & 1.06 & 1.19 & 1.55 \\
\hline 2.5 & 1.66 & 2.30 & 3.07 & 1.13 & 1.40 & 1.65 & 1.01 & 1.03 & 1.11 \\
\hline 3.0 & 1.28 & 1.67 & 2.07 & 1.04 & 1.16 & 1.27 & 1.00 & 1.01 & 1.02 \\
\hline 3.5 & 1.14 & 1.40 & 1.63 & 1.02 & 1.07 & 1.12 & 1.00 & 1.00 & 1.00 \\
\hline 4.0 & 1.08 & 1.25 & 1.40 & 1.01 & 1.03 & 1.06 & 1.00 & 1.00 & 1.00 \\
\hline
\end{tabular}

TABLE 8: Developed gamma control chart ARLs comparison with existing control charts when $\mathrm{ARL}_{0}=500$ and $m=5$.

\begin{tabular}{|c|c|c|c|c|c|c|c|c|c|}
\hline$s$ & $\begin{array}{c}\text { Proposed } \\
k_{1}=3.3615 \\
k_{2}=1.5835\end{array}$ & $\begin{array}{c}\alpha_{0}=5 \\
\text { MDS } \\
k_{1}=4.4575 \\
k_{2}=2.3070\end{array}$ & $\begin{array}{c}\text { Shewhart } \\
L=3.0458\end{array}$ & $\begin{array}{c}\text { Proposed } \\
k_{1}=3.3245 \\
k_{2}=1.6015\end{array}$ & $\begin{array}{c}\alpha_{0}=10 \\
\text { MDS } \\
k_{1}=4.5285 \\
k_{2}=2.3135\end{array}$ & $\begin{array}{c}\text { Shewhart } \\
L=3.0701\end{array}$ & $\begin{array}{c}\text { Proposed } \\
k_{1}=3.4165 \\
k_{2}=1.5815\end{array}$ & $\begin{array}{c}\alpha_{0}=20 \\
\text { MDS } \\
k_{1}=4.5120 \\
k_{2}=2.3165\end{array}$ & $\begin{array}{c}\text { Shewhart } \\
L=3.0804\end{array}$ \\
\hline 1.0 & 500.01 & 500.05 & 500.94 & 500.04 & 500.05 & 500.93 & 500.05 & 500.05 & 500.04 \\
\hline 1.1 & 263.92 & 268.09 & 283.07 & 215.54 & 218.55 & 244.74 & 143.46 & 151.15 & 185.05 \\
\hline 1.2 & 117.99 & 121.35 & 144.60 & 69.52 & 72.53 & 100.63 & 27.60 & 33.80 & 57.41 \\
\hline 1.3 & 54.22 & 57.08 & 78.26 & 24.82 & 27.61 & 46.63 & 8.08 & 10.89 & 22.64 \\
\hline 1.4 & 27.29 & 29.77 & 46.15 & 10.76 & 12.89 & 24.79 & 3.58 & 5.02 & 11.04 \\
\hline 1.5 & 15.24 & 17.31 & 29.45 & 5.67 & 7.22 & 14.78 & 2.11 & 3.01 & 6.33 \\
\hline 1.6 & 9.39 & 11.09 & 20.09 & 3.52 & 4.66 & 9.66 & 1.55 & 2.15 & 4.12 \\
\hline 1.7 & 6.31 & 7.70 & 14.47 & 2.49 & 3.36 & 6.80 & 1.31 & 1.72 & 2.96 \\
\hline 1.8 & 4.56 & 5.71 & 10.91 & 1.95 & 2.63 & 5.08 & 1.18 & 1.47 & 2.29 \\
\hline 1.9 & 3.50 & 4.48 & 8.54 & 1.63 & 2.18 & 3.98 & 1.11 & 1.32 & 1.88 \\
\hline 2.0 & 2.83 & 3.66 & 6.90 & 1.44 & 1.89 & 3.24 & 1.07 & 1.22 & 1.61 \\
\hline 2.5 & 1.56 & 2.01 & 3.29 & 1.11 & 1.29 & 1.72 & 1.01 & 1.04 & 1.12 \\
\hline 3.0 & 1.25 & 1.52 & 2.17 & 1.04 & 1.11 & 1.29 & 1.00 & 1.01 & 1.03 \\
\hline 3.5 & 1.14 & 1.31 & 1.69 & 1.02 & 1.05 & 1.14 & 1.00 & 1.00 & 1.01 \\
\hline 4.0 & 1.08 & 1.20 & 1.44 & 1.01 & 1.02 & 1.07 & 1.00 & 1.00 & 1.00 \\
\hline
\end{tabular}

(1) The out-of-control ARL and SDRL values decline speedily when the shift $(s)$ of the manufacturing process increases

(2) It is detected that the chart coefficient $k_{2}$ shows an increasing tendency for increased value of $k$ for a fixed value of $m$ when other parametric combinations are fixed

(3) From the tables, it is noticed that $\mathrm{ARL}_{1}$ and SDRL values decrease when $m$ values increase. In addition,
$\mathrm{ARL}_{1}$ and SDRL values increased with the increase of $k$ value (i.e., $m-2$ to $m$ - 0 ). It also observed the same inclination over the other parametric combinations and $\mathrm{ARL}_{0}=370$ and 500

(4) It is interesting to observe from the results that the values of $\mathrm{ARL}_{1}$ and SDRL are small for $k=m-2$ and these values are increasing from $k=m-2$ to $k=m$ for fixed values of $m$. In addition, noticed that $\mathrm{ARL}_{1}$ and SDRL values are large at $k=m$ as compared to the values at $k=m-1$ and $k=m-2$ (we know that 

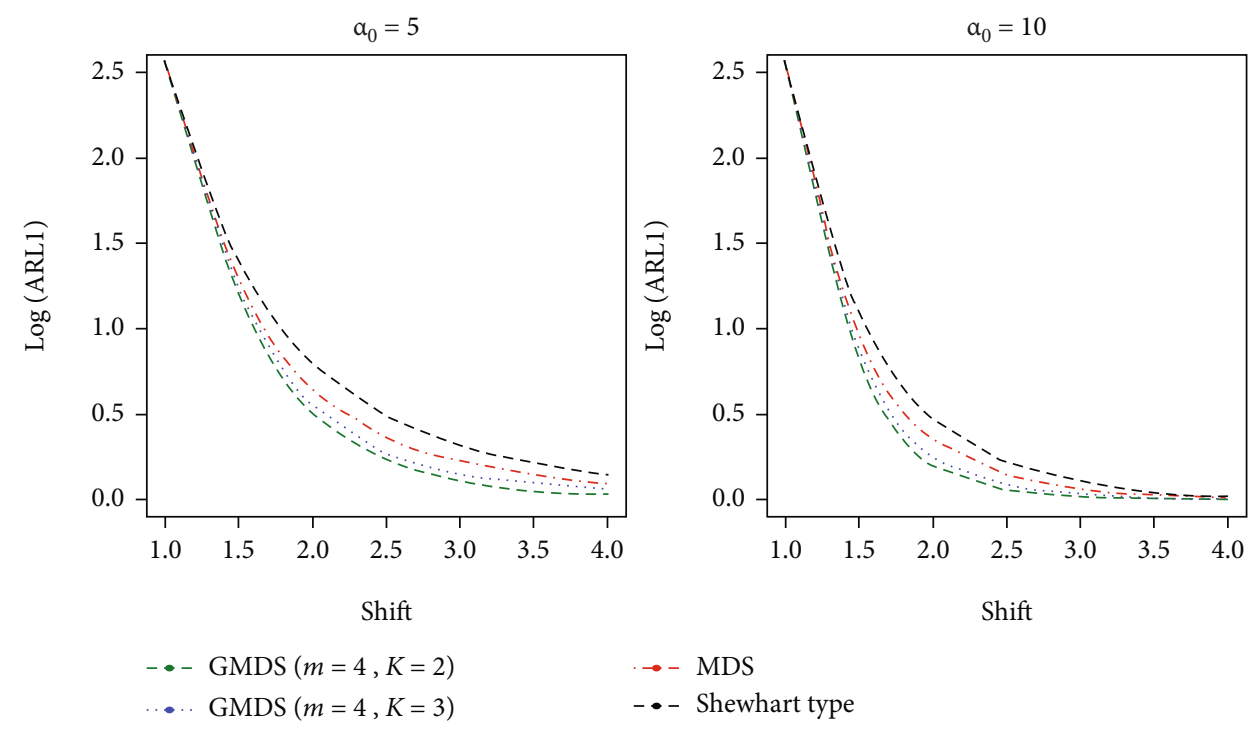

Figure 1: ARL curves of gamma control chart for three charts for $m=4$ and $\mathrm{ARL}_{0}=370$.
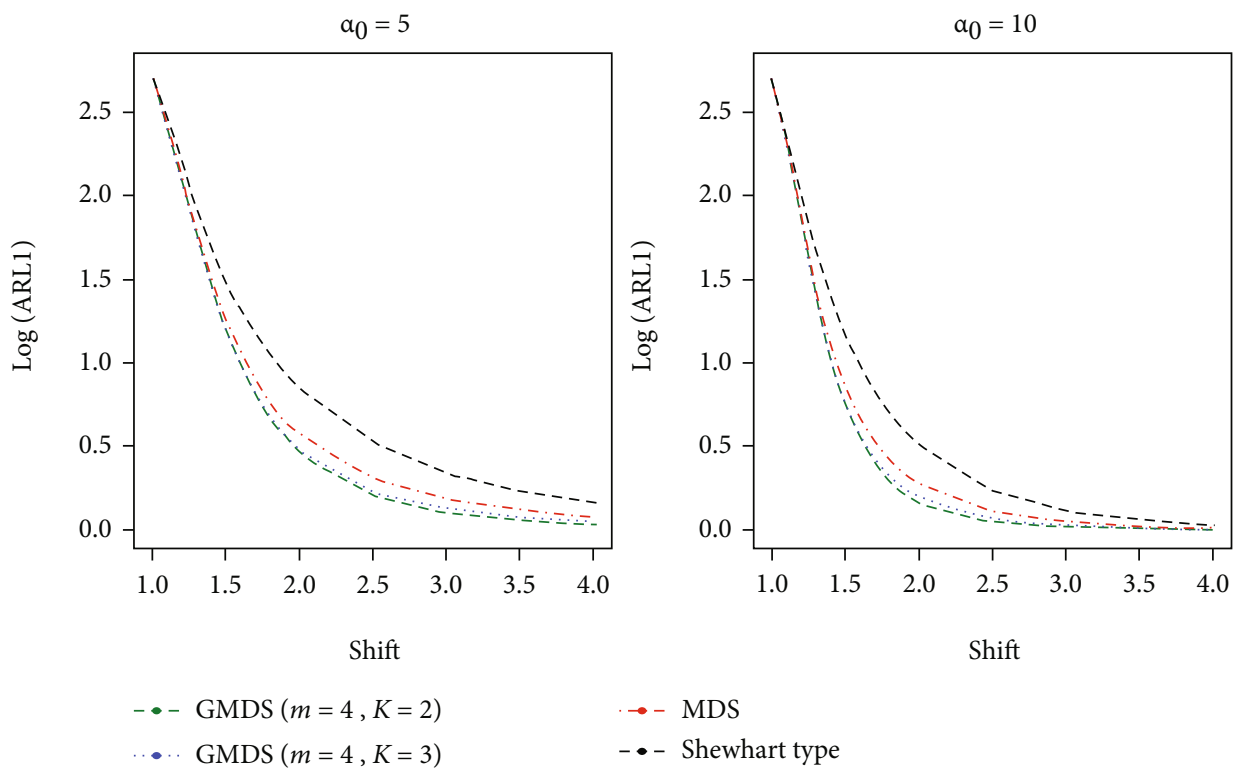

FIGURE 2: ARL curves of gamma control chart for three charts for $m=5$ and $\mathrm{ARL}_{0}=500$.

if $k=m$, the developed plan becomes MDS design). Hence, it is concluded from the results that gamma control chart using GMDS sampling is an enormous amount of accurate than gamma control chart using MDS sampling

\section{Comparison with Existing Charts}

In this part, a comparison is made between the developed control chart and the existing Shewhart type control chat and MDS control chart for gamma distribution. Also, the application of developed control chart and its dominance over available control chart schemes studied using real data set is presented. In addition, through a simulation study, the supremacy of the developed control chart when com- pared with the existing control charts is examined. The performance of the developed control chart is studied through ARL values and we know that a control chart with smaller ARL values is more desirable. In this investigation, we studied when $\mathrm{ARL}_{0}=370$ and $\mathrm{ARL}_{0}=500$; the shape parameter of gamma distribution is given as $\alpha_{0}=5,10$, and 20 to compare the developed gamma control chart under GMDS with the existing MDS and Shewhart type control chart at various shift values. These comparisons are presented in Table 7 for $\mathrm{ARL}_{0}=370$ and $m=4$ and in Table 8 for $\mathrm{ARL}_{0}=500$ and $m=5$ at various shape parameters of the gamma distribution.

It is noticed that from the results on the basis of Tables 7 and 8 , the developed gamma control charts show smaller quantity $\mathrm{ARL}_{1}$ values as compared with the MDS and Shewhart type control charts at various shifts $(s)$ values and 
TABLE 9: The simulated data when $m=5, \alpha_{0}=5$, and $\mathrm{ARL}_{0}=500$.

\begin{tabular}{lcccccccccccc}
\hline S. no. & $X$ & $X^{*}$ & S. no. & $X$ & $X^{*}$ & S. no. & $X$ & $X^{*}$ & S. no. & $X$ & $X^{*}$ \\
\hline 1 & 7.6063 & 1.9666 & 16 & 2.3608 & 1.3315 & 31 & 8.0437 & 2.0036 & 46 & 4.6261 & 1.6662 \\
2 & 2.8743 & 1.4218 & 17 & 2.5800 & 1.3715 & 32 & 4.6680 & 1.6713 & 47 & 5.4935 & 1.7645 \\
3 & 3.2301 & 1.4782 & 18 & 4.9637 & 1.7058 & 33 & 8.6453 & 2.0524 & 48 & 1.9999 & 1.2599 \\
4 & 4.6671 & 1.6712 & 19 & 4.1035 & 1.6010 & 34 & 9.1395 & 2.0908 & 49 & 6.4479 & 1.8613 \\
5 & 7.0398 & 1.9165 & 20 & 2.7647 & 1.4035 & 35 & 4.0200 & 1.5900 & 50 & 11.1001 & 2.2307 \\
6 & 4.3621 & 1.6339 & 21 & 3.8728 & 1.5704 & 36 & 6.2734 & 1.8443 & 51 & 7.4944 & 1.9569 \\
7 & 3.5145 & 1.5204 & 22 & 8.0095 & 2.0008 & 37 & 2.7584 & 1.4024 & 52 & 8.1311 & 2.0109 \\
8 & 7.8831 & 1.9902 & 23 & 8.3195 & 2.0263 & 38 & 6.4997 & 1.8662 & 53 & 3.4348 & 1.5088 \\
9 & 7.2328 & 1.9339 & 24 & 3.2821 & 1.4861 & 39 & 7.6433 & 1.9698 & 54 & 3.2860 & 1.4867 \\
10 & 7.0640 & 1.9187 & 25 & 5.5956 & 1.7753 & 40 & 10.1004 & 2.1616 & 55 & 4.8631 & 1.6942 \\
11 & 3.7153 & 1.5488 & 26 & 3.4608 & 1.5126 & 41 & 11.0929 & 2.2302 & 56 & 7.6722 & 1.9723 \\
12 & 5.0059 & 1.7106 & 27 & 4.2462 & 1.6193 & 42 & 5.9905 & 1.8162 & 57 & 6.2898 & 1.8459 \\
13 & 2.6249 & 1.3794 & 28 & 6.6523 & 1.8807 & 43 & 3.9466 & 1.5803 & 58 & 6.1469 & 1.8318 \\
14 & 3.6922 & 1.5456 & 29 & 6.5704 & 1.8730 & 44 & 9.1140 & 2.0888 & 59 & 9.3127 & 2.1039 \\
15 & 4.5475 & 1.6567 & 30 & 5.2560 & 1.7387 & 45 & 11.0067 & 2.2244 & 60 & 3.2213 & 1.4769 \\
\hline
\end{tabular}

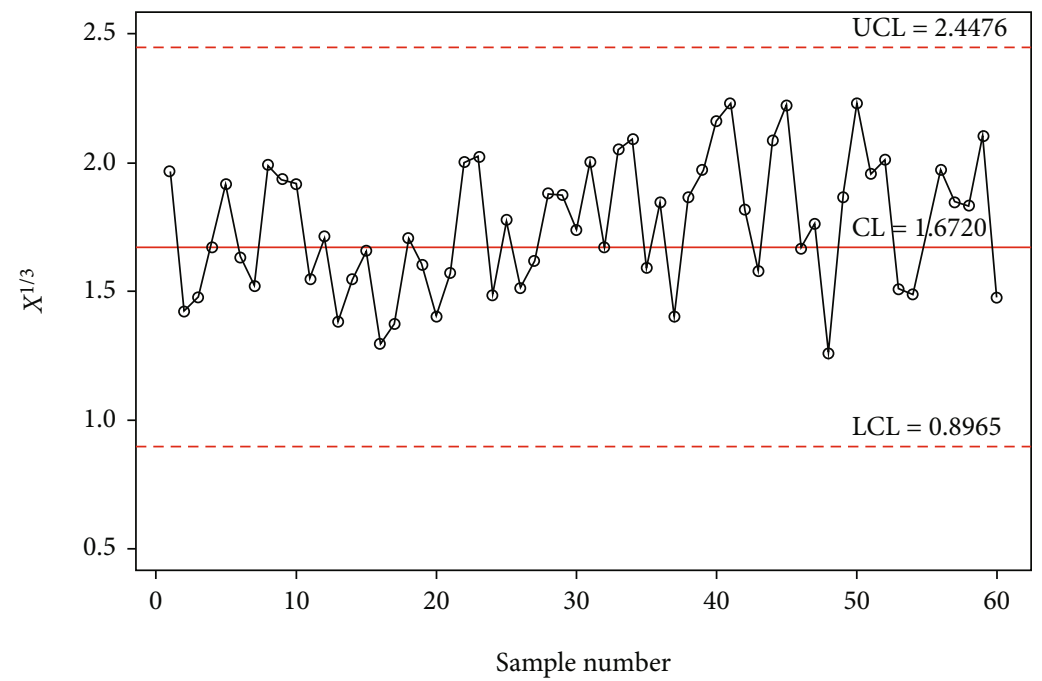

FIgURe 3: Gamma control chart for Shewhart type for simulated data when $m=5, \alpha_{0}=5$, and $\mathrm{ARL}_{0}=500$.

various parametric values studied in this article. At a glance, when $\mathrm{ARL}_{0}=370, \alpha_{0}=5$ and $s=1.4$ from Table 7 , for the developed control chart $\mathrm{ARL}_{1}=27.17$ whereas $\mathrm{ARL}_{1}=$ 31.10 for MDS scheme and $\mathrm{ARL}_{1}=38.44$ from the Shewhart type control chart. Similarly, for $\mathrm{ARL}_{0}=500, \alpha_{0}=10$, and $s$ $=1.5$ from Table 8 , we sense that the developed control chart gives $\mathrm{ARL}_{1}=5.67$ while $\mathrm{ARL}_{1}=7.22$ for the MDS control chart and $\mathrm{ARL}_{1}=14.28$ from the Shewhart type control chart. The graphical presentation is given to show the performance of developed control chat over the existing MDS and Shewhart type control charts along with various shift values (see Figures 1 and 2). From these two figures, it is articulated that the developed gamma control chart based on GMDS is certified extra sensitive as compared to the MDS and the Shewhart-type control charts. To draw attention to this con- clusion, a real data illustration and a simulation study are also carried out in the following subsections.

4.1. Simulation Analysis. In order to investigate the implementation of the planned control chart over the available control charts, a simulation study is conducted. In this investigation, 30 samples are generated from the gamma distribution with shape parameter $\alpha_{0}=5$ and in-control scale parameter 1 and last 30 random samples are generated from a gamma distribution with shape parameter $\alpha_{0}=5$ and outof-control scale parameter 1.4 (i.e., the shift of $s=1.4$ ). The data is reported in Table 9 alongside computed statistic $X_{i}^{*}$ $=X_{i}^{1 / 3}$. The control chart coefficients at $m=5, \alpha_{0}=5$, and $\mathrm{ARL}_{0}=500$ are available in Table 8. The Shewhart type gamma control chart is given in Figure 3, and MDS gamma 


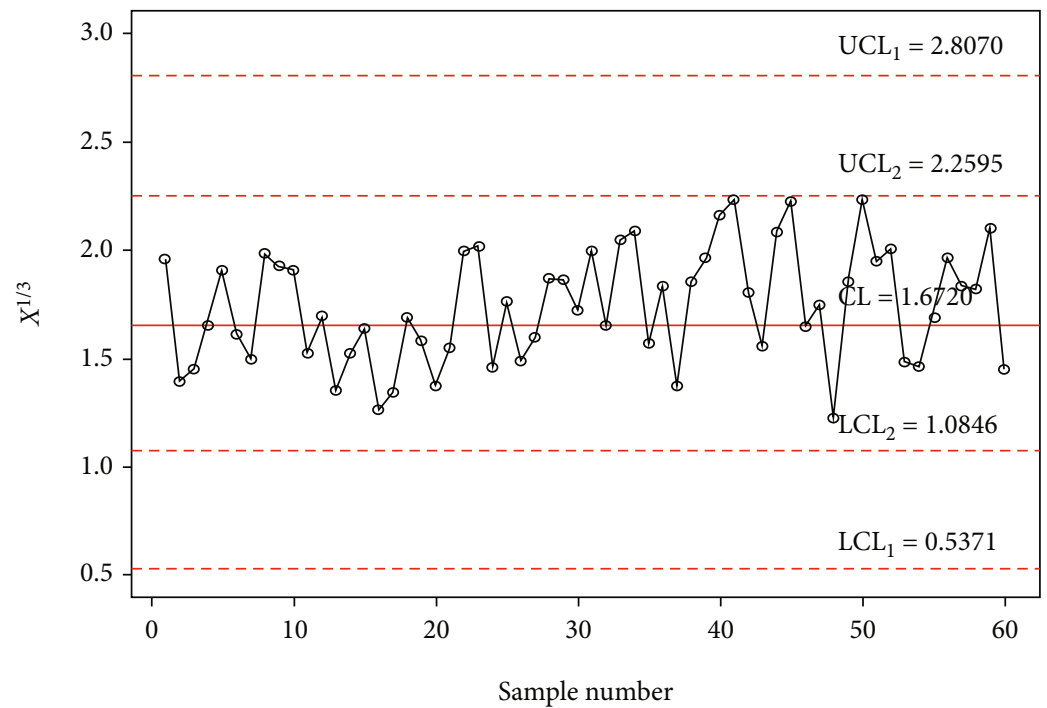

FIGURE 4: Gamma control chart using MDS sampling for simulated data when $m=5, \alpha_{0}=5$, and $\mathrm{ARL}_{0}=500$.

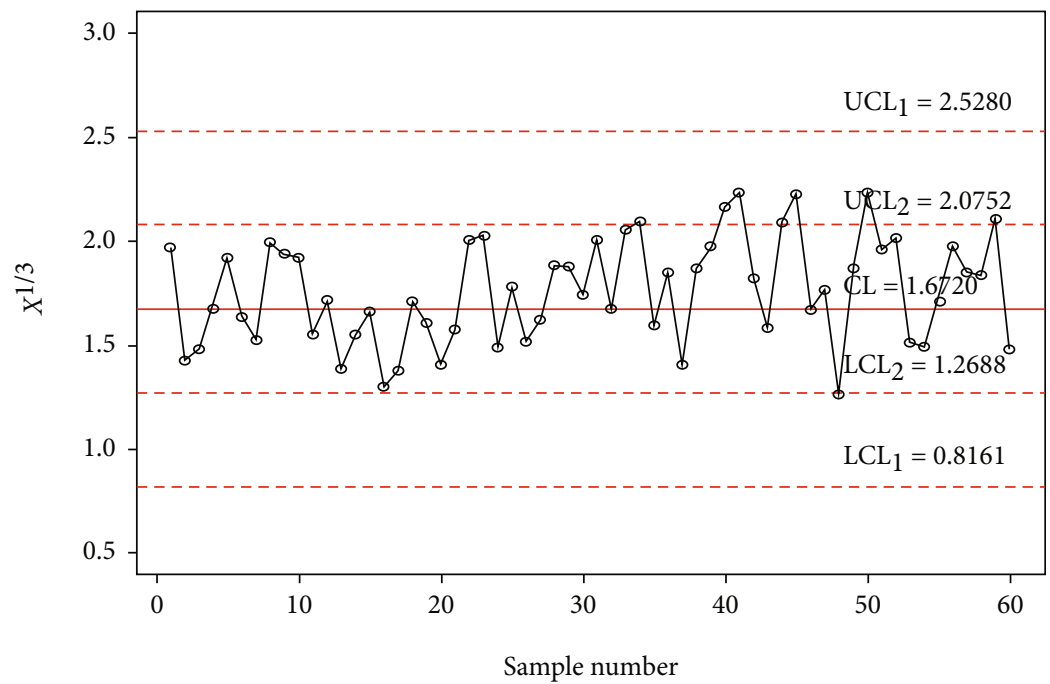

Figure 5: Gamma control chart using GMDS sampling for simulated data when $m=5, \alpha_{0}=5$, and $\mathrm{ARL}_{0}=500$.

control chart when $m=5$ and $k=5$ is provided in Figure 4. The gamma control chart using GMDS sampling when $m$ $=5$ and $k=3$ is depicted in Figure 5 . According to the above scheme for gamma control charts, we implement the MDS chart as follows: if previous 5 (since $m=5$ ) $X_{i}^{*}$ values are displayed between the inner control limits, then the process is considered to be under control while for proposed gamma control chart under GMDS, the process is said to be declared as under control if no less than 3 out of 5 previous (since $k=3$ and $m=5) X_{i}^{*}$ values are within the interior control limits.

From Figures 3 and 4, it is apparent that the gamma control charts based on Shewhart type and MDS scheme are unable to notice the shift. On the other hand, in Figure 5, it can be found that using gamma control charts under the GMDS scheme detects out-of-control at sample numbers 34, 40, 41, 44, 45, 50, and 59. Present simulation examines that the developed gamma control chart based on GMDS sampling is more efficient than the gamma control charts as compared to MDS and Shewhart type design.

4.2. Application of the Proposed Chart for COVID-19 Data. In the present section, the developed gamma control chart using GMDS sampling is applied to the monitoring of Coronavirus (COVID-19) outbreak in China. The data set is borrowed from Li et al. [38], and they discussed mortality caused by COVID-19 based on collected data of 33 death cases in Wuhan city of Hubei province during an early outbreak as well as confirmed cases and death toll. They have studied the COVID-19 outbreak in China by considering some specific regions as representative samples. The days from ICU intake to death caused by COVID-19 data are presented in Table 10 along with the transformation of the variable. To 
TABLE 10: Death cause by COVID-19 in Wuhan city of Hubei province in China.

\begin{tabular}{lccccc}
\hline Subgroup no. & Days from ICU intake to death $(X)$ & $X^{*}$ & Subgroup no. & Days from ICU intake to death $(X)$ & $X^{*}$ \\
\hline 1 & 4 & 1.5874 & 18 & 17 & 2.5713 \\
2 & 6 & 1.8171 & 19 & 5 & 1.7100 \\
3 & 5 & 1.7100 & 20 & 8 & 2.0000 \\
4 & 7 & 1.9129 & 21 & 8 & 2.0000 \\
5 & 5 & 1.7100 & 22 & 8 & 2.0000 \\
6 & 4 & 1.5874 & 23 & 1 & 1.0000 \\
7 & 2 & 1.2599 & 24 & 12 & 2.2894 \\
8 & 6 & 1.8171 & 25 & 10 & 2.1544 \\
9 & 10 & 2.1544 & 26 & 12 & 2.2894 \\
10 & 1 & 1.0000 & 27 & 4 & 1.5874 \\
11 & 7 & 1.9129 & 28 & 4 & 1.5874 \\
12 & 9 & 2.0801 & 29 & 2 & 1.2599 \\
13 & 22 & 2.8020 & 30 & 4 & 1.5874 \\
14 & 11 & 31 & 1 & 1.0000 \\
15 & 6 & 32240 & 32 & 25 & 2.2240 \\
16 & 8 & 1.8171 & & & 2.9240 \\
17 & 14 & 2.0000 & 33 & & \\
\hline
\end{tabular}


Figure 6: The empirical and theoretical cdfs and Q-Q plots for the GD for the days from ICU intake to death.

fit the gamma distribution, the parameters are estimated using the maximum likelihood approach, and the shape is $2.0026 \approx 2.0$, and the scale parameter is 3.9185. The Kolmogorov-Smirnov test is 0.1197 , and $p$ value is 0.7322 . Figure 6 displays the histogram and Q-Q plot to highlight the goodness of fit of the gamma distribution. Hence, gamma distribution furnishes a good fit for the days from ICU intake to death caused by COVID-19 data.

The chart constants at the estimated shape parameter of 2 are obtained using a simulation procedure given in Section 2 . The control chart constants at $\alpha_{0}=2$ and $m=4$ are $k_{1}=$ 3.1035 and $k_{2}=1.4645$ for a developed control chart, $k_{1}$ $=3.7525$ and $k_{2}=2.1935$ for MDS control chart, and $L=$ 2.8828 for Shewhart type control chart. The chart limits of the developed control chart, MDS, and Shewhart type control charts for days from ICU intake to death data are given in Figures 7-9. In Figures 7-9, the proposed control charts for GMDS, MDS, and Shewhart type control charts are displayed by plotting the control limits and chat statistics $X^{*}$. Using days from ICU intake to death data, the developed control chart can be exemplified as follows: declare the process as in-control when 4 earlier values of $X^{*}$ fall in the inner control limits of the developed gamma control chart using the MDS plan. While in the case of the developed gamma control chart using GMDS scheme, the process can be expected as in-control when at least 2 out of 4 earlier values of $X^{*}$ fall in the inner control limits.

It is clearly noticeable from Figure 7 that all sample statistics are inside the upper and lower control limits. Hence, it shows that Shewhart-type gamma control chart fails to detect a change in the process. On the other hand, if we consider gamma control chart, using MDS design also failed to identify changes in the process due to all sample statistics inside the inner upper and inner lower control limits (see Figure 8), whereas the gamma control chart based on GMDS design experiences the out-of-control signals at sample numbers 10 , 




FIGURE 7: Shewhart type gamma control chart for days from ICU intake to death data.

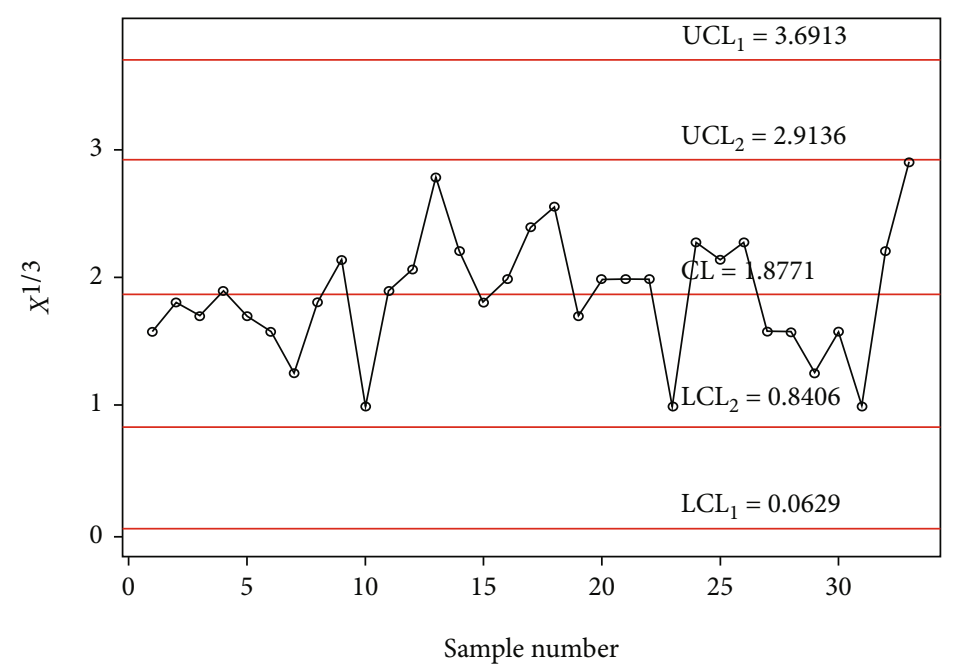

FIGURE 8: Gamma control chart using MDS sampling for days from ICU intake to death data.

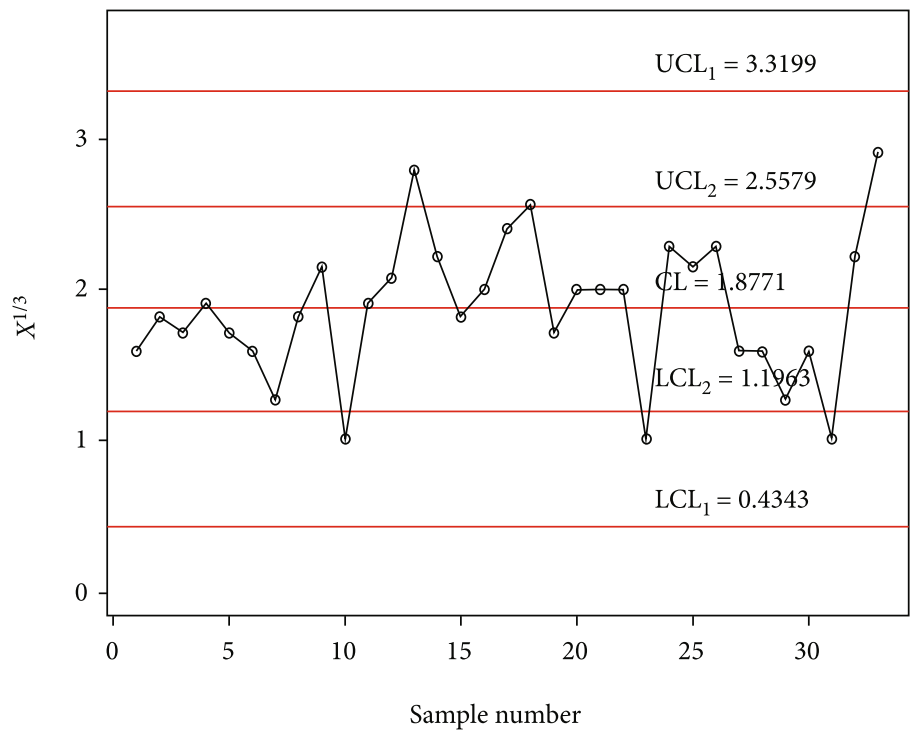

FIGURE 9: Gamma control chart using GMDS sampling for days from ICU intake to death data. 
$13,23,31$, and 33, respectively (see Figure 9). Therefore, from the methodology explained in Section 2, it reveals that the developed gamma control chart based on GMDS design is faster in detecting process variation as compared with the existing control chart based MDS and Shewhart type. Hence, from real application in the field of medical sciences, data for days from ICU intake to death revels that the gamma control chart based on GMDS remains superior methodology as compared to the existing control charts considered in this study.

\section{Conclusions}

We developed the gamma control chart based on GMDS sampling. The computational methodology is also discussed for ARL and SDRL when the process is in-control and out-ofcontrol. The control chart parameters for the proposed control chart are obtained using in-control average run length $\left(\mathrm{ARL}_{0}\right)$ and out-of-control ARL $\left(\mathrm{ARL}_{1}\right)$ of the proposed gamma control chart using GMDS sampling. The performance of the proposed control chart is investigated using simulation for the various shifts in the scale parameter. Tables of chart parameters alongside out-of-control ARL $\left(\mathrm{ARL}_{1}\right)$ for various shift values for specified shape parameters are displayed. Furthermore, a comparative study is also carried out with the developed gamma control chart based on GMDS sampling over the existing MDS and Shewhart type gamma control charts using the ARLs. The results display that the developed gamma control chart based on GMDS sampling shows reduced $A R L_{1}$ values as compared with the existing two gamma control chart discussed in this article.

The implementation of the developed control chart is demonstrated using simulation study as well as tangible data from ICU intake to death cause by COVID-19 and it is shown that designed gamma control chart using GMDS sampling detected out-of-control samples whereas the MDS and Shewhart type gamma control charts failed to detect the outof-control signal. Hence, we conclude that the gamma control chart based on GMDS is a superior methodology as compared to the existing control charts considered in this study to detect a shift in the parameter. The developed control chart method in this paper can be used in different industrial and medical situations specifically when the researcher would like to discover a small and moderate shift in quality characteristics. Future research maybe considered as control charts for some nonnormal distributions and cost consideration using GMDS sampling design.

\section{Appendix}

R Coding to Run the Algorithm

\#\# compute mean and standard deviation

$\mathrm{mts}<-\mathrm{b}^{\wedge}(1 / 3) * \operatorname{gamma}(\mathrm{a}+1 / 3) / \mathrm{gamma}(\mathrm{a})$

vts $<-b^{\wedge}(2 / 3) *$ gamma $(a+2 / 3) / g a m m a(a)-m_{t} \wedge 2$

sdts $<$-sqrt(vts)

\#\# fixing the range of $\mathrm{k} 1$ and $\mathrm{k} 2$

$\mathrm{q} 1<-\operatorname{seq}(2.61,6.96$, by $=0.0005)$

$\mathrm{q} 2<-$ seq $(1.21,6.86$, by $=0.0005)$

$\mathrm{vv}<-$ gamma $(\mathrm{a}+2 / 3) / \operatorname{gamma}(\mathrm{a})-(\operatorname{gamma}(\mathrm{a}+1 / 3)$

/gamma(a))^2



choose those $\mathrm{k} 1$ and $\mathrm{k} 2$ values

\section{Data Availability}

The data is given in the paper.

\section{Conflicts of Interest}

The authors declare that they have no conflicts of interest.

\section{Acknowledgments}

This article was funded by the Deanship of Scientific Research (DSR) at King Abdulaziz University, Jeddah. The authors therefore acknowledge DSR with thanks for the technical and financial support. The work by Chi-Hyuck Jun was supported by Korea Institute for Advancement of Technology (KIAT) grant funded by the Korea Government (MSIT) (N0008691, the Competency Development Program for Industry Specialist).

\section{References}

[1] D. C. Montgomery, Statistical Quality Control: A Modern Introduction, John Wiley \& Sons, Inc., Hoboken, New Jersey, 7th edition, 2013.

[2] N. F. Zhang, "Detection capability of residual control chart for stationary process data," Journal of Applied Statistics, vol. 24, no. 4, pp. 475-492, 1997.

[3] Y. C. Lin and C. Y. Chou, "Robustness of the variable sample size and control limit XChart to non normality," Communications in Statistics Theory and Methods, vol. 34, no. 3, pp. 721743, 2005.

[4] M. Haynes, K. Mengersen, and P. Rippon, "Generalized control charts for non-normal data using $g$-and- $k$ distributions," Communications in Statistics-Simulation and Computation, vol. 37, no. 9, pp. 1881-1903, 2008.

[5] E. Santiago and J. Smith, "Control charts based on the exponential distribution: adapting runs rules for thetChart," Quality Engineering, vol. 25, no. 2, pp. 85-96, 2013. 
[6] H. Aksoy, "Use of gamma distribution in hydrological analysis," Turkish Journal of Engineering and Environmental Sciences, vol. 24, no. 6, pp. 419-428, 2000.

[7] H. A. al-Oraini and M. Rahim, "Economic statistical design of $\mathrm{X}$ control charts for systems with $\operatorname{gamma}(\lambda, 2)$ in-control times," Computers \& Industrial Engineering, vol. 43, no. 3, pp. 645-654, 2002.

[8] D. Jearkpaporn, D. C. Montgomery, G. C. Runger, and C. M. Borror, "Process monitoring for correlated gammadistributed data using generalized-linear-model-based control charts," Quality and Reliability Engineering International, vol. 19, no. 6, pp. 477-491, 2003.

[9] S. H. Sheu and T. C. Lin, "The generally weighted moving average control chart for detecting small shifts in the process mean," Quality Engineering, vol. 16, no. 2, pp. 209-231, 2003.

[10] M. Aslam, M. Azam, N. Khan, and C.-H. Jun, "A control chart for an exponential distribution using multiple dependent state sampling," Quality \& Quantity, vol. 49, no. 2, pp. 455-462, 2015.

[11] C. W. Zhang, M. Xie, J. Y. Liu, and T. N. Goh, “A control chart for the gamma distribution as a model of time between events," International Journal of Production Research, vol. 45, no. 23, pp. 5649-5666, 2007.

[12] N. L. Johnson and S. Kotz, Distributions in Statistics: Continuous Univariate Distributions: Vol. 2, Houghton Mifflin, 1970.

[13] L. S. Nelson, "A control chart for parts-per-million nonconforming items," Journal of Quality Technology, vol. 26, no. 3, pp. 239-240, 1994.

[14] M. A. Mohammed, "Using statistical process control to improve the quality of health care," Quality and Safety in Health Care, vol. 13, no. 4, pp. 243-245, 2004.

[15] M. A. Mohammed and D. Laney, "Overdispersion in health care performance data: Laney's approach," Quality and Safety in Health Care, vol. 15, no. 5, pp. 383-384, 2006.

[16] M. Aslam, M. Mohsin, and C.-H. Jun, "A new t-chart using process capability index," Communications in Statistics-Simulation and Computation, vol. 46, no. 7, pp. 5141-5150, 2017.

[17] A. W. Wortham and R. C. Baker, "Multiple deferred state sampling inspection," International Journal of Production Research, vol. 14, no. 6, pp. 719-731, 1976.

[18] S. Balamurali and C.-H. Jun, "Multiple dependent state sampling plans for lot acceptance based on measurement data," European Journal of Operational Research, vol. 180, no. 3, pp. 1221-1230, 2007.

[19] M. Aslam, O. H. Arif, and C.-H. Jun, "A control chart for gamma distribution using multiple dependent state sampling," Industrial Engineering and Management Systems, vol. 16, no. 1, pp. 109-117, 2017.

[20] R. Vaerst, "A procedure to construct multiple deferred state sampling plan," Methods of Operations Research, vol. 37, pp. 477-485, 1982.

[21] V. Soundararajan and R. Vijayaraghavan, "On designing multiple deferred state sampling (MDS-1 $(0,2))$ plans involving minimum risks," Journal of Applied Statistics, vol. 16, no. 1, pp. 87-94, 1989.

[22] V. Soundararajan and R. Vijayaraghavan, "Construction and selection of multiple dependent (deferred) state sampling plan," Journal of Applied Statistics, vol. 17, no. 3, pp. 397409, 1990.

[23] V. Kuralmani and K. Govlndaraju, "Selection of multiple deferred (dependent) state sampling plans," Communications in Statistics-Theory and Methods, vol. 21, no. 5, pp. 13391366, 1992.

[24] K. Govindaraju and K. Subramani, "Selection of multiple deferred (dependent) state sampling plans for given acceptable quality level and limiting quality level," Journal of Applied Statistics, vol. 20, no. 3, pp. 423-428, 1993.

[25] M. Aslam, M. Azam, and C.-H. Jun, "Multiple dependent state sampling plan based on process capability index," Journal of Testing and Evaluation, vol. 41, no. 2, article 20120012, 2013.

[26] M. Aslam, C.-H. Yen, C.-H. Chang, and C.-H. Jun, "Multiple dependent state variable sampling plans with process loss consideration," International Journal of Advanced Manufacturing Technology, vol. 71, no. 5-8, pp. 1337-1343, 2014.

[27] M. Aslam, A. Nazir, and C.-H. Jun, "A new attribute control chart using multiple dependent state sampling," Transactions of the Institute of Measurement and Control, vol. 37, no. 4, pp. 569-576, 2015.

[28] M. Aslam, S. Balamurali, M. Azam, G. S. Rao, and C. H. Jun, "Mixed multiple dependent state sampling plans based on process capability index," Journal of Testing and Evaluation, vol. 43, no. 1, article 20130009, 2015.

[29] M. Aslam, L. Ahmad, C.-H. Jun, and O. H. Arif, “A control chart for COM-Poisson distribution using multiple dependent state sampling," Quality and Reliability Engineering International, vol. 32, no. 8, pp. 2803-2812, 2016.

[30] M. Aslam, F.-K. Wang, N. Khan, and C.-H. Jun, "A multiple dependent state repetitive sampling plan for linear profiles," Journal of the Operational Research Society, vol. 69, no. 3, pp. 467-473, 2018.

[31] C.-H. Yen, C.-H. Chang, M. Aslam, and C.-H. Jun, "Multiple dependent state repetitive sampling plans based on one-sided process capability indices," Communications in StatisticsTheory and Methods, vol. 47, no. 6, pp. 1403-1412, 2018.

[32] M. A. Raza and M. Aslam, "Design of control charts for multivariate Poisson distribution using generalized multiple dependent state sampling," Quality Technology and Quantitative Management, vol. 16, no. 6, pp. 629-650, 2019.

[33] G. S. Rao, M. Aslam, and C. H. Jun, “A variable sampling plan using generalized multiple dependent state based on a onesided process capability index," Communications in StatisticsSimulation and Computation, pp. 1-12, 2019.

[34] G. S. Rao, M. A. Raza, M. Aslam, A. H. al-Marshadi, and C. H. Jun, "A variable control chart based on process capability index under generalized multiple dependent state sampling," IEEE Access, vol. 7, pp. 34031-34044, 2019.

[35] M. Aslam, S. Balamurali, and C. H. Jun, "A new multiple dependent state sampling plan based on the process capability index," Communications in Statistics-Simulation and Computation, pp. 1-17, 2019.

[36] M. Aslam, M. A. Raza, and C. H. Jun, "A new variable control chart under generalized multiple dependent state sampling," Communications in Statistics-Simulation and Computation, vol. 49, no. 9, pp. 2321-2332, 2020.

[37] E. B. Wilson and M. M. Hilferty, "The distribution of chisquare," Proceedings of the National Academy of Sciences of the United States of America, vol. 17, no. 12, pp. 684-688, 1931.

[38] J. Li, L. Wang, S. Guo et al., "The data set for patient information based algorithm to predict mortality cause by COVID19," Data in Brief, vol. 30, article 105619, 2020. 